\title{
Novel insights into a treatment for aplastic anemia based on the advanced proliferation of bone marrow-derived mesenchymal stem cells induced by fibroblast growth factor 1
}

\author{
SHAYI JIANG, MIN XIA, JINGWEI YANG, JINGBO SHAO, XUELIAN LIAO, JIASHI ZHU and HUI JIANG \\ Department of Hematology, Shanghai Children's Hospital, Shanghai Jiaotong University, \\ Shanghai 200040, P.R. China
}

Received October 31, 2014; Accepted July 21, 2015

DOI: $10.3892 / \mathrm{mmr} .2015 .4421$

\begin{abstract}
Aplastic anemia (AA) is rare disease that is predominantly observed in adolescents. Without effective management at an early stage, is associated with a high risk of mortality. Bone marrow mesenchymal stem cells (BMSCs) can differentiate into various types of cell, which are able to produce a number of hematopoietic growth factors considered to be important in AA alleviation. However, the mechanism underlying the role of fibroblast growth factor 1 (FGF1) in BMSC differentiation remains unknown. In the current study, the investigation focused on the regulatory role and potential signaling pathway of FGF1 in BMSC differentiation in patients exhibiting AA. BMSCs were infected with Ad-FGF1 and presented a potent proliferation capability, which was evaluated using Cell Counting kit- 8 analysis. Reverse transcription-quantitative polymerase chain reaction revealed that long non-coding (lnc)RNA of testis development related gene 1 (TDRG1) was significantly upregulated, demonstrating high expression at the transcriptional level in the BMSCs that were infected with Ad-FGF1. The decreased proliferation capability of BMSCs that were treated with Ad-FGF1 and TDRG1-small interfering RNA validated the vital effect of TDRG1 on the FGF1 regulatory process of BMSC differentiation. Further experiments revealed that the increase of acetyl-histones, $\mathrm{H} 3$ and $\mathrm{H} 4$ was diminished in the TDRG1 promoter of BMSCs that were infected with Ad-FGF1, which indicated that the process of acetylation was promoted when the BMSCs were infected with Ad-FGF1. Thus, it was inferred that FGF1 induces the proliferation of BMSCs in patients with AA via promoting acetylation in lncRNA of the TDRG1 gene promoter.
\end{abstract}

Correspondence to: Dr Hui Jiang, Department of Hematology, Shanghai Children's Hospital, Shanghai Jiaotong University, No. 24, Lane 1400 West Beijing Road, Shanghai 200040, P.R. China E-mail: hjiang_1@163.com

Key words: fibroblast growth factor 1, bone marrow mesenchymal stem cells, long non-coding RNA, aplastic anemia

\section{Introduction}

Aplastic anemia (AA), a rare bone marrow disease, which leads to pancytopenia, anemia, leukopenia and thrombocytopenia mainly occurs in teenagers worldwide (1). Although the pathogenesis of AA has been associated with chemicals, drugs (2), radiation (3), infection and immune diseases (4), the precise cause remains unknown in half of the cases of AA (5). Without effective treatment, AA is associated with a high risk of mortality (6), therefore, studies regarding the underlying pathogenesis are considered to be necessary and relevant.

Bone marrow mesenchymal stem cells (BMSCs) are multipotent stromal cells, which differentiate into numerous types of cell, such as osteoblasts, chondrocytes and adipocytes (7). The capability to support hematopoiesis and immunomodulatory characteristics render BMSCs vital in the bone marrow hematopoietic microenvironment (8). Since the abnormal alteration of BMSCs was observed in patients with AA (9), various studies have investigated the association between BMSCs and the pathogenesis of AA. Zhao et al (10) showed that AA BMSCs were prone to differentiate into adipocytes rather than osteoblasts. However, treatment with arsenic trioxide partially reversed the differentiation imbalance. Wang et al (11) treated BMSCs (obtained from patients with AA) with rapamycin at varying concentrations and identified that rapamycin was vital in the suppression of BMSC proliferation, cell cycle progression and adipogenesis (8). However, the underlying mechanisms of the influence of BMSCs on AA treatment by activating growth factor remains unclear and require further investigation.

Recently, the investigation of AA-associated BMSC differentiation at the gene level has become increasingly prevalent. Jiang et al (12) demonstrated that basic fibroblastic growth factor (FGF) was expressed at a low level in the BMSCs of infants presenting with AA and subsequently inferred that low FGF expression may be involved in the pathogenesis of AA. FGFs, are a family of pluripotent growth factors that affect mitosis, cell regulation and morphology, as well as the endocrine system. Thus far, 22 members of the FGF family have been identified and verified to be structurally associated with molecular signaling (13). Furthermore, FGF1, encoded by FGF1, exerts potent activity on cell survival, embryonic 
development, as well as tissue repair (14). Stegmann (15) identified that FGF-1 promoted neoangiogenesis in the hypoxic heart muscle of humans and demonstrated the angiogenic effect of FGF-1. Cao et al (16) reported that FGF1 and FGF2 exhibited more potent efficacy on angiogenesis compared with vascular endothelial or platelet-derived growth factors, and induced the formation of stable vascular networks. On the basis of previous research, the aim of the present study was to investigate the regulatory mechanism of FGF1 in BMSCs to provide a novel insight into the management of AA.

Long non-coding (lnc) RNAs are non-protein coding transcripts which contain $>200$ nucleotides (17). lncRNAs have been reported to be significant in dosage compensation effects, the regulation of epigenetics, the cell cycle and cell differentiation in mammals (18). Due to their unknown, but potentially efficacious applications, researchers worldwide have focused on establishing databases of lncRNAs at a genome-wide level (19). Thus far, the constructed lncRNA databases are as follows: IncRNABase (20), ChIPBase (21), LNCipedia (22), lncRNAdb (23), NONCODE (24) and NRED (25).

In the present study, the potential association between FGF1 and BMSCs in patients with AA was investigated, and the regulatory mechanism of FGF1 by lncRNAs was evaluated to provide a novel insight into the treatment of AA.

\section{Materials and methods}

Isolation and culture of BMSCs. Marrow was obtained from patients diagnosed with aplastic anemia (AA), which had been preserved in the Cancer Tissue Bank between 2007 and 2013 at Changzhou First People's Hospital (Jiangsu, China). Among the 24 selected tumor samples, 12 were from male patients and 12 were from female patients. The average age of the patients was 36 years. Informed consent for the experimental use of surgical samples was obtained from all patients. The study was approved by the ethics committee of The First People's Hospital of Changzhou, Changzhou, China. Following heparinization $(3,000$ units; $0.2 \mathrm{ml}), 1 \mathrm{ml}$ of marrow, was added to $5 \mathrm{ml}$ Red Blood Cell Lysis Buffer (Beyotime Institute of Biotechnology, Nanjing, China) and the homogeneous mixture was centrifuged at $2,930 \mathrm{x}$ g for $10 \mathrm{~min}$. The supernatant was discarded and the precipitate was rinsed twice with phosphate-buffered saline ( $\mathrm{pH}$ 7.2). The BMSCs were isolated by an additional centrifugation of the mixture and isometric percoll lymphocyte separation medium (Ficoll-lsopaque, Pharmacia, Piscataway, NJ, USA) $(\varrho=1.072 \mathrm{~g} / \mathrm{ml})$ was added. The mixture was cultured in $\alpha$-minimum essential medium ( $\alpha$-MEM) supplemented with $10 \%$ fetal bovine serum (FBS), $100 \mathrm{U} / \mathrm{ml}$ penicillin (Sigma-Aldrich, St. Louis, MO, USA) and $100 \mathrm{U} / \mathrm{ml}$ streptomycin (Sigma-Aldrich) at $37^{\circ} \mathrm{C}$ with $5 \% \mathrm{CO}_{2}$ for $24 \mathrm{~h}$. Finally, the isolated BMSCs were subcultured every 3 days according to whether the ratio of the original medium to the fresh medium was $1: 2(\mathrm{v}: \mathrm{v})$.

Reconstruction of the adenovirus vector. The pSileneerl.0-shFGF1 and pShuttle vectors (BD Biosciences, Palo Alto, CA, USA) were cut using BamHI and HindIII restriction enzymes (Promega Corporation, Madison, WI, USA). Then the fragments of shFGF1 cDNA $(0.3 \mathrm{~kb})$ and pShuttle $(4.2 \mathrm{~kb})$ were retrieved and ligated using T4 DNA ligase for
$4 \mathrm{~h}$ at $22^{\circ} \mathrm{C}$. DH5 $\alpha^{\mathrm{TM}}$ competent cells were transformed and the plasmids were extracted following screening for positive colonies in Luria-Bertani (LB) medium supplemented with kanamycin. The combination of the materials was termed pShuttle-shFGF1 To construct the recombinant adenovirus vector, cells were transfected with pAdxsi vector as well as pShuttle-shFGF1. Superstratum was covered with 5\% gelose and cultured at $37^{\circ} \mathrm{C}$ with $5 \% \mathrm{CO}_{2}$ for 10 days. Following connection of the retrieved plasmids and fragments with their target genes [FGF1 or small interfering (si)RNA-testis development related gene 1 (TDRG1), designed and synthesized by Invitrogen Life Technologies (San Diego, CA, USA)], the DH5 $\alpha$ was transformed and coated onto LB medium with ampicillin (Marsan Pharmaceuticals, Cherry Hill, NJ, USA). The positive colonies were selected by sending to Sigma-Aldrich for sequencing. The cultured BMSCs $\left(5 \times 10^{5} /\right.$ well) were seeded into a 6-well plate filled with Dulbecco's modified Eagle's medium (DMEM; Life Technologies, Inc., Gaithersburg, MD, USA) and infected using Lipofectamine 2000 (Invitrogen Life Technologies, Carlsbad, CA, USA) until the cells covered $80-90 \%$ of the plate. Two days later, the virus was collected using the cytopathic effect and the titer was determined with a hemolytic plaque assay using the following model: Virus titer $=$ No. of plaques/dilution factor $\mathrm{x}$ volume of diluent. For comparison, a sham control (medium only) was included and underwent the above-mentioned procedure. All culture processes were conducted in an atmosphere of $5 \% \mathrm{CO}_{2}$ at $37^{\circ} \mathrm{C}$ and the experiments were performed in triplicate.

Cell proliferation assay. The suspension of BMSCs, FGF1-BMSCs, TDRG1 siRNA-BMSCs, FGF1-TDRG1 siRNA-BMSCs, scramble siRNA-BMSCs and FGF1-scramble siRNA-BMSCs were plated in 96-well plates at a concentration of $100 \mathrm{ml} /$ well in $\alpha$-MEM with $10 \%$ FBS. The cells were then cultured by incubation at $37^{\circ} \mathrm{C}$ in a $5 \% \mathrm{CO}_{2}$ atmosphere for 24, 36, 48, 60 and 72 h. Cell Counting kit-8 (CCK-8; Dojindo Laboratory, Kumamoto, Japan) reagent $(10 \mathrm{ml})$ was added to the well and incubated for 24, 36, 48, 60 or $72 \mathrm{~h}$. After a 2-h incubation, the optical density (OD) values of corresponding cells were measured using an ultraviolet spectrophotometer (Varian Medical Systems, Inc., Palo Alto, CA, USA) at $450 \mathrm{~nm}$. The results were recorded for further comparison.

Immunofluorescence. After being fixed on a 48-well plate using $4 \%$ paraformaldehyde, the infected cells were permeabilized using $0.2 \%$ Triton X-100 and then sealed using 5\% goat serum for $30 \mathrm{~min}$. Incubation with anti-FGF1 primary antibody (Abcam,Cambridge, MA,USA; cat.no.ab9588; dilution, 1:200) and a fluorescein isothiocyanate (FITC)-labeled secondary antibody (cat. no. ABIN101988; Upstate Biotechnology, Lake Placid, NY, USA) was conducted at $37^{\circ} \mathrm{C}$. The cell nucleus was counterstained with 4',6-diamino-2-phenylindole and the plate was sealed using glycerinum. Microscopy (Olympus IX71, Tokyo, Japan) was performed to observe and obtain images the cells.

Reverse transcription-quantitative polymerase chain reaction (RT-qPCR). To investigate the expression of target genes, total RNA was extracted and isolated from BMSCs using TRIzol reagent (Invitrogen Life Technologies). RNA was 
reverse transcribed using M-MLV Reverse Transcriptase (Promega Corporation). RNA quality was assessed with the ThermoScientific NanoDrop1000 (Thermo Fisher Scientific, Inc., Waltham, MA, USA). RT-qPCR was performed using the QuantiTect Primer assay (Qiagen GmbH, Hilden, Germany) and QuantiTect SYBR Green RT-PCR kit (Qiagen GmbH) on a LightCycler 480 Instrument (Roche Diagnostics, Mannheim, Germany). The detection and quantification contained the following steps: Reverse transcription was performed for $30 \mathrm{~min}$ at $55^{\circ} \mathrm{C}$ and initial activation for $15 \mathrm{~min}$ at $95^{\circ} \mathrm{C}$; followed by 40 cycles of denaturation conducted at $94^{\circ} \mathrm{C}$ for $15 \mathrm{sec}$, annealing for $30 \mathrm{sec}$ at $55^{\circ} \mathrm{C}$ and extension for $30 \mathrm{sec}$ at $72^{\circ} \mathrm{C}$. The target gene primers were designed by Invitrogen Life Technologies and primer sequences were as follows: Forward: 5'-CAGTACTTGGCCATGGACA-3' and reverse: 5'-AGTGAGTCCGAGGACCGC-3'. The outcome of the RT-qPCR was assessed using the $2^{-\Delta \Delta C t}$ method and GAPDH served as a reference for normalizing the target gene expression.

Chromatin immunoprecipitation (ChIP). BMSCs were cross-linked with $1 \%$ formaldehyde for $10 \mathrm{~min}$ at room temperature. The cross-linking was terminated by adding $125 \mathrm{mM}$ glycine and the cells were washed twice with ice-cold PBS. The cells were solubilized in a buffer containing $10 \mathrm{mM}$ Tris- $\mathrm{HCl}$ (pH 8.0), 1\% Triton X-100, 1\% sodium deoxycholate, $1 \mathrm{mM}$ phenylmethanesulfonyl fluoride and protease inhibitor cocktail for $10 \mathrm{~min}$ at $4^{\circ} \mathrm{C}$. Sonication using a Bioruptor ${ }^{\circledR}$ Sonicator (Diagenode s.a., Seraing, Belgium) was performed to shear chromatin into 500-bp fragments. The supernatant was obtained by centrifugation $\left(16,000 \mathrm{x}\right.$ g for $10 \mathrm{~min}$ at $\left.4^{\circ} \mathrm{C}\right)$ and equally divided into six tubes (100 $\mu \mathrm{l} /$ tube). The appropriate antibody [anti-histone deacetylase (HDAC) 3 (Abcam; cat. no. ab7030; dilution: 1:500) or HDAC4 (Abcam; ab53331; dilution, 1:500] was added into each tube and incubated for $3 \mathrm{~h}$ at $4^{\circ} \mathrm{C}$. Immunoprecipitation was performed using ChIP-grade agarose beads with protein $\mathrm{G}$ (Cell Signaling Technology, Inc., Danvers, MA, USA), and the cells were blocked with $1 \%$ bovine albumin and $1 \%$ salmon sperm DNA. Finally, ChIP-grade agarose beads, protein G, cells, bovine albumin and salmon sperm DNA, were collected and the DNA was isolated by sedimentation velocity for qPCR.

Statistical analysis. Data were processed using SPSS 12.0 statistical software (SPSS, Inc., Chicago, IL, USA) and recorded as the mean \pm standard error of the mean. $\mathrm{P}<0.05$ was considered to indicate a statistically significant difference. In addition, one-way analysis of variance was adopted to assess the data. All of the experiments were performed in triplicate for the purposes of comparison.

\section{Results}

FGF1 promotes the proliferation of BMSCs. The expression of FGF1 was identified in the BMSCs by immunofluorescence and the cells infected with Ad-FGF1 were observed to divide vigorously (Fig. 1A and B). According to the results of the CCK-8 assay in Fig. 1C, BMSCs of patients with AA infected with FGF1 grew more markedly when compared with the non-treated BMSCs (control group) with significantly higher
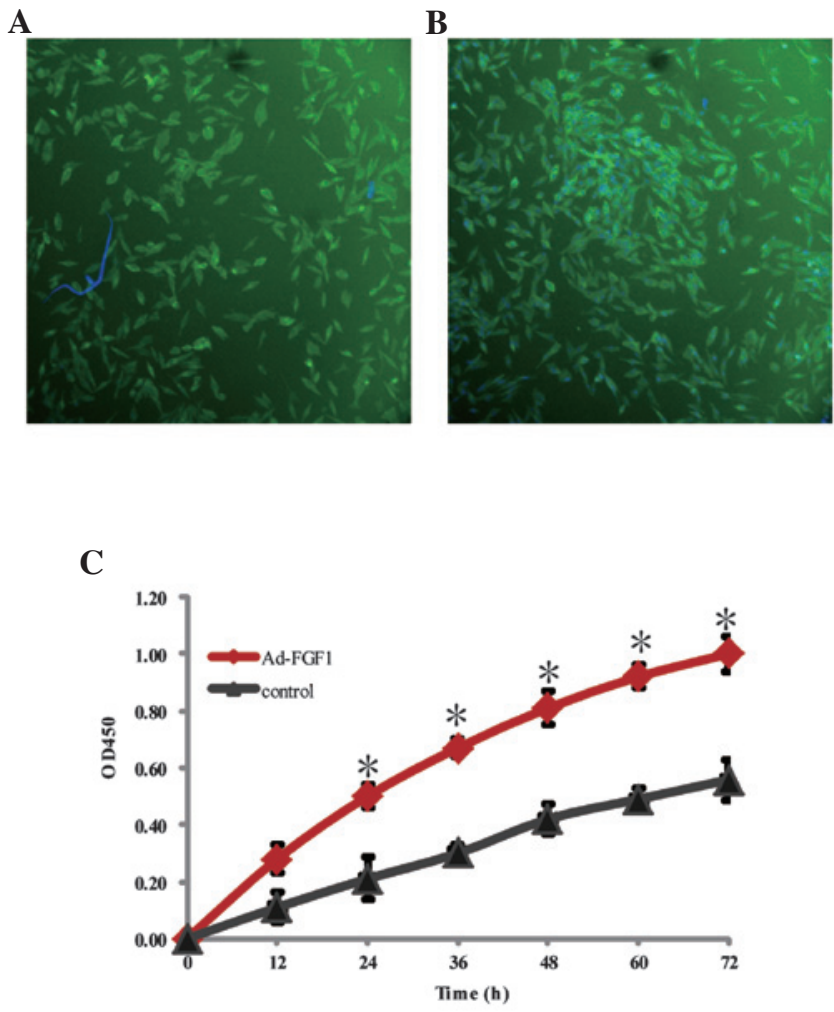

Figure 1. Effects of FGF1 on BMSC proliferation. The FGF1 expression and efficacy in BMSCs were observed under a microscope on the basis of immunofluorescence. (A) Control group of BMSCs. (B) BMSCs infected with FGF1. Magnification, x200. (C) The capability of proliferation was assessed using Cell Counting kit-8. The plots of OD values for BMSCs and FGF1-BMSCs are presented for comparison. The data are presented as the mean \pm standard error of the mean from three independent experiments. ${ }^{*} \mathrm{P}<0.01$ compared with the OD450 value in the control group. FGF1, fibroblast growth factor 1; BMSC, bone marrow mesenchymal stem cell; OD, optical density.

OD values from $24 \mathrm{~h}$ during culture. Therefore, it was inferred that FGF1 may promote the proliferation of BMSCs in patients with AA.

FGF1 influences the expression of IncRNA TDRG1. The expression levels of IncRNAs in BMSCs infected with/without Ad-FGF1 were detected by RT-qPCR (Fig. 2). The upregulated expression of all of the selected genes was observed in the two groups. Compared with the control group, the genes in Ad-FGF1-infected BMSCs were observed to be upregulated and expressed at higher levels. The two genes that were significantly upregulated compared with the control group were TDRG1 and urothelial carcinoma-associated 1 (UCA1; $\mathrm{P}<0.05)$, which indicated that the infection with Ad-FGF1 may impact the proliferation of BMSCs by influencing lncRNA. In order to confirm the result described above, the expression level of TDRG1 was determined by RT-qPCR. The expression of TDRG1 was observed to be significantly higher in BMSCs infected with Ad-FGF1 than that in the control group (Fig. 3A; $\mathrm{P}<0.05)$. This finding illustrates that FGF1 influences the expression of lncRNA TDRG1.

FGF1 promotes acetylation in the TDRG1 gene promoter. HDAC3 and HDAC4, which are members of the histone deacetylase/acetylase family was encoded by HDAC3 and 


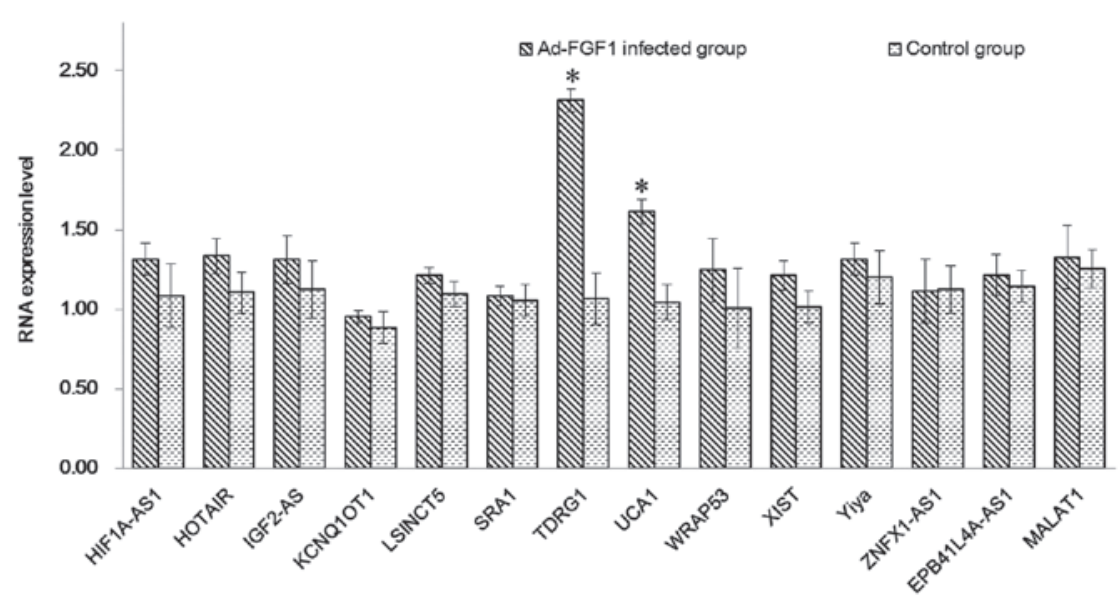

Figure 2. Expression level of disease-associated lncRNA in BMSCs and FGF1-BMSCs. The total RNA was extracted following infection with/without an adenovirus vector containing the FGF1 gene. Analysis of lncRNA expression was subsequently performed via reverse transcription-quantitative polymerase chain reaction. The data are presented as the mean \pm standard error of the mean from three independent experiments. ${ }^{*} \mathrm{P}<0.05$, compared with control. FGF1, fibroblast growth factor 1; HIF1a-AS1, hypoxia inducible factor 1-antisense RNA 1; TDRG1, testis development related gene 1; UCA1, urothelial carcinoma-associated 1; MALAT1, metastasis associated lung adenocarcinoma transcript 1; lncRNA, long non-coding RNA; BMSC, bone marrow mesenchymal stem cell.

A
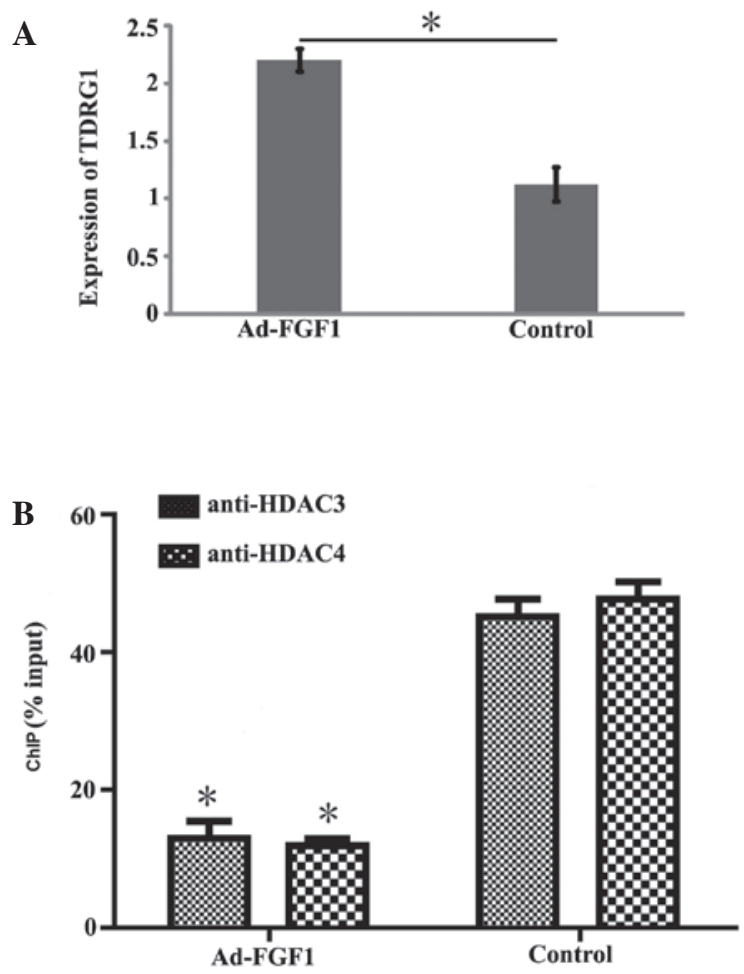

Figure 3. Deacetylation level of lncRNA TDRG1 gene varied in BMSCs overexpressing FGF1. (A) Total RNA was extracted following infection with/without an adenovirus vector containing the FGF1 gene and analysis of lncRNA TDRG1 expression was performed via reverse transcription-quantitative polymerase chain reaction. " $\mathrm{P}<0.05$ compared with the control group. (B) The deacetylation level of the lncRNA TDRG1 gene was analyzed by ChIP sequencing. ${ }^{*} \mathrm{P}<0.01$ compared with the the control group. Data are presented as the mean \pm standard error of the mean from three independent experiments. FGF1, fibroblast growth factor 1; HDAC, histone deacetylase; ChIP, Chromatin immunoprecipitation; lncRNA, long non-coding RNA; BMSC, bone marrow mesenchymal stem cell; TDRG1, testis development related gene 1 .

HDAC4, respectively. HDAC 3 and HDAC4 possess deacetylase activity and regulate the process of acetylation/deacetylation in order to alter chromosome structure and impact the access

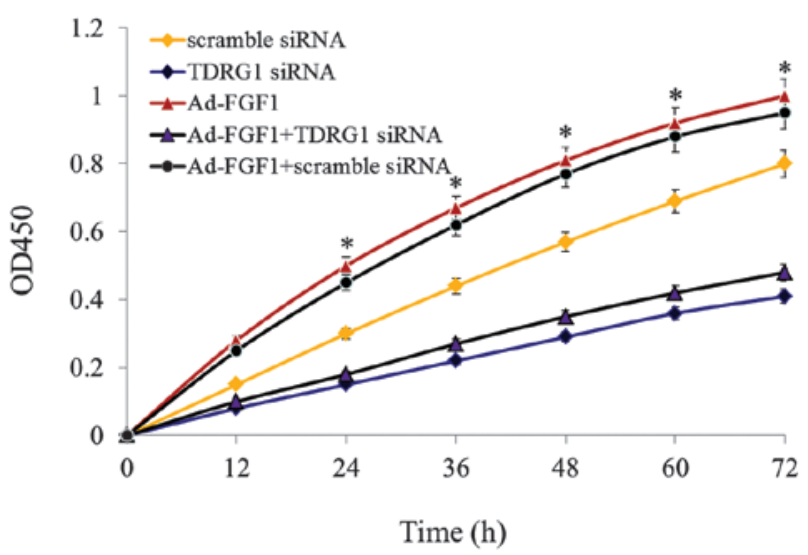

Figure 4. FGF1 enhances the proliferation of bone marrow mesenchymal stem cells through lncRNA TDRG1. The effect of IncRNA TDRG1 on cell proliferation was evaluated by RNA interference. Scramble siRNA served as a negative control. The data are presented as the mean \pm standard error of the mean from three independent experiments. "P $<0.05$ compared with the TDRG1 siRNA group. si, small interfering; TDRG1, testis development related gene 1; FGF1, fibroblast growth factor 1; OD, optical density.

of transcription factor to DNA. Therefore, it was hypothesized that the acetylation level on the promoter region of the TDRG1 gene may be regulated by the overexpression of FGF1 in BMSCs. This hypothesis was tested via ChIP experiments using anti-HDAC 3 and anti-HDAC4 antibodies. As shown in Fig. 3B, the deacetylation level of the TDRG1 gene promoter was significantly reduced in BMSCs overexpressing FGF1 when compared with the control group, which indicates that decreased enrichment of HDAC 3 and HDAC4 results in the overexpression of FGF1, leading to acetylation of the TDRG1 gene promoter.

FGF1 enhances the proliferation of BMSCs through lncRNA $T D R G 1$. In order to analyze the function of TDRG1 lncRNA, an RNA interference molecule was designed and TDRG1 lncRNA was silenced in BMSCs infected with/without Ad-FGF1. The 
results are presented in Fig. 4. Following TDRG1 lncRNA silencing, cell proliferation was observed to be significantly downregulated in BMSCs that were infected with/without Ad-FGF1, when compared with the control groups $(\mathrm{P}<0.05)$. All of these results indicate that FGF1 enhances the proliferation of BMSCs via regulating the expression of TDRG1 lncRNA.

\section{Discussion}

AA is a rare disease in which the normal generation of red blood cells and various white cell lines in the bone marrow is disrupted $(26,27)$. Various studies have focused on investigating the pathogenesis of AA by observing the regulation of gene expression levels. Zhang et al (28) demonstrated that the increased expression of $\mathrm{T}$ cell immunoglobulin and immunoreceptor tyrosine-based inhibition motif domain ameliorated immune-mediated bone marrow failure of AA via the depressed function of $\mathrm{CD}^{+} \mathrm{T}$ cells. Furthermore, Wang et al (29) confirmed the harmful and protective function of human leukocyte antigen alleles in Chinese patients exhibiting severe AA. In the current study, it was inferred that FGF1 is a potential regulator of BMSC differentiation in patients with AA on the basis of experiments in which low expression of FGF was observed the BMSCs of patients with AA. As BMSCs are significant in the hematopoietic microenvironment, an investigation concerning the underlying mechanism of FGF1 in the differentiation of BMSCs was considered to be essential and necessary.

The association between FGF1 and BMSCs has been reported in various studies. Eom et al (30) demonstrated that FGF4 and FGF2 exerted an autocrine effect by regulating the proliferation of BMSCs. Chen et al (31) demonstrated that the mutation of Ser252Trp in FGF receptor 2 was vital in FGF signaling and reduced the proliferation of BMSCs. In the present study, research was initiated with the study of the association between FGF1 and BMSC proliferation. The increased OD value of the cultured FGF1-BMSCs (when compared with the control group) revealed the increased proliferative capability of the cells infected with Ad-FGF1 and illustrated that FGF1 was a potential regulator of BMSC proliferation. Furthermore, this finding was supported by results of immunofluorescence in which vigorous proliferation and FGF1 expression were observed in FGF1-BMSCs.

In recent years, lncRNA has become increasingly popular in the investigation of the pathogenesis of certain malignant diseases. Metastasis associated lung adenocarcinoma transcript 1 (MALAT1) and UCA1 were found to be abnormally expressed in melanoma metastases (32). X-inactive specific transcript was identified as a biomarker for membranous nephropathy (33) and WD repeat containing, antisense to TP53 was considered as a candidate for a cancer susceptibility gene (34). The bi-functional lncRNA, steroid receptor activator RNA 1 was identified to be a component of the steroid receptor coactivator-1 acetyltransferase complex and indicated to be an epigenetic regulatory component (35). Insulin-like growth factor 2 antisense RNA was observed to be a potent stimulator in cancer cell lines of diabetic patients (36). Furthermore, HOX transcript antisense RNA and MALAT1 were identified as the specific lncRNAs in laryngeal squamous cell carcinoma (37).
The overexpression of hypoxia inducible factor 1-antisense RNA 1 (HIF1A-AS1) was associated with non-papillary renal HIF1A carcinomas (38). The disease-related lncRNA of erythrocyte membrane protein band 4.1 like 4A-AS1, zinc finger, NFX1-type containing 1-AS1, Yiya and long stress-induced non-coding transcript 5 were selected from lncRNABase together with the above-mentioned lncRNAs, and determined in the present study using RT-qPCR to observe the potential regulatory role of IncRNAs in FGF1-BMSCs. According to the findings of the PCR, all the genes were observed to be upregulated in the FGF1-BMSCs; however, two genes (TDRG1 and UCA1) exhibited significant increases in expression. It was indicted that FGF1 promoted BMSC proliferation via regulation of the IncRNAs of TDRG1 and UCA1.

Although numerous studies have documented their association with certain diseases, investigations involving the role of TDRG1 and UCA1 in Ad-FGF1-infected BMSCs are considered to be limited. According to the result of Fig. 3, the expression of TDRG1 at the gene level in FGF1-BMSCs increased significantly compared with control cells. When the cells were infected with TDRG1 siRNA, proliferation was markedly inhibited, this further confirmed the role of TDRG1 in BMSC differentiation in patients with AA. In Fig. 4, the expression of HDAC3 and HDAC4 (indicators of acetylation levels in cells) was observed to be significantly reduced in FGF1-BSMCs. Since HDAC3 and HDAC4 possess the potent activity of deacetylation, a decrease in their expression levels would lead to enhanced acetylation, which introduces the acetyl functional group into a chemical compound and is considered to be an important modification process of proteins in cell biology.

Therefore, it was concluded in the present study that FGF1 promotes the proliferation of BMSCs to alleviate AA by enhancing acetylation of the IncRNA of the TDRG1 gene promoter.

The findings of the current study provide a promising insight into the therapeutic treatment of AA. However, further validation via in vivo studies is required.

\section{Acknowledgements}

The present study was supported by Shanghai Jiaotong University School of Medicine Science Fund Project (grant no. 2013236).

\section{References}

1. Sheng W, Liu C, Fu R, Wang H, Qu W, Ruan E, Wang G, Liu H, $\mathrm{Wu}$ Y, Song J, et al: Abnormalities of quantities and functions of linker for activations of T cells in severe aplastic anemia. Eur J Haemato 93: 214-223, 2014.

2. Young NS, Scheinberg P and Calado RT. Aplastic anemia. Current Opin Hematol 15: 162-168, 2008.

3. Cohen $\mathrm{T}$ and Creger WP. Acute myeloid leukemia following seven years of aplastic anemia induced by chloramphenicol. Am J Med 43: 762-770, 1967.

4. Binder D, van den Broek MF, Kägi D, Bluethmann H, Fehr J, Hengartner $\mathrm{H}$ and Zinkernagel RM: Aplastic anemia rescued by exhaustion of cytokine-secreting CD8+ T cells in persistent infection with lymphocytic choriomeningitis virus. J Exp Med 187: 1903-1920, 1998.

5. Xing L, Liu C, Fu R, Wang H, Wang J, Liu X, Feng L, Li L, Liu H, Wang H, et al: CD8+HLA-DR+ T cells are increased in patients with severe aplastic anemia. Mol Med Rep 10: 1252-1258, 2014. 
6. Feng X, Scheinberg P, Biancotto A, Rios O, Donaldson S, Wu C, Zheng H, Sato K, Townsley DM, McCoy JP and Young NS: In vivo effects of horse and rabbit antithymocyte globulin in patients with severe aplastic anemia. Haematologica 99: 1433-1440, 2014.

7. Kato RB, Roy B, De Oliveira FS, Ferraz EP, De Oliveira PT, Kemper AG, Hassan MQ, Rosa AL and Beloti MM: Nanotopography directs mesenchymal stem cells to osteoblast lineage through regulation of microRNA-SMAD-BMP-2 circuit. J cell Physiol 229: 1690-1696, 2014.

8. Ding L, Zhu H, Yang Y, Wang ZD, Zheng XL, Yan HM, Dong L, Zhang HH, Han DM, Xue M, et al: Functional mesenchymal stem cells remain present in bone marrow microenvironment of patients with leukemia post-allogeneic hematopoietic stem cell transplant. Leuk lymphoma 55: 1635-1644, 2014.

9. Sun ZM, Liu HL, Geng LQ, Wang XB, Yao W, Liu X, Ding KY, Han YS, Yang HZ, Tang BL, et al: HLA-matched sibling transplantation with G-CSF mobilized PBSCs and BM decreases GVHD in adult patients with severe aplastic anemia. J Hematol Oncol 3: 51, 2010.

10. Zhao J, Wang C, Song Y and Fang B: Arsenic trioxide and microRNA-204 display contrary effects on regulating adipogenic and osteogenic differentiation of mesenchymal stem cells in aplastic anemia. Acta Biochim Biophys Sin (Shanghai) 46: 885-893, 2014

11. Wang X, Ma FX, Lu SH, Chi Y, Chen F, Li X, Li JJ, Du WJ, Feng Y, Cui JJ, et al: Effects of rapamycin on biological characteristics of bone marrow mesenchymal stem cells from patients with aplastic anemia. Zhongguo Shi Yan Xue Ye Xue Za Zhi 22 762-766, 2014 (In Chinese).

12. Jiang SY, Xie XT, Jiang H, Zhou J, Li FX and Cao P: Low expression of basic fibroblastic growth factor in mesenchyma stem cells and bone marrow of children with aplastic anemia. Pediatric Hematol Oncol 31: 11-19, 2014.

13. Tursi A, Elisei W, Inchingolo CD, Nenna R, Picchio M, Ierardi E and Brandimarte G: Chronic diverticulitis and Crohn's disease share the same expression of basic fibroblastic growth factor syndecan 1 and tumour necrosis factor- $\alpha$. J Clin Pathol 67 : 844-846, 2014

14. Marinucci L, Bodo M, Balloni S, Locci P and Baroni T: Sub-toxic nicotine concentrations affect extracellular matrix and growth factor signaling gene expressions in human osteoblasts. J Cell Physiol 229: 2038-2048, 2014

15. Stegmann TJ: New approaches to coronary heart disease: Induction of neovascularisation by growth factors. Bio Drugs 11 301-308, 1999.

16. Cao R, Bråkenhielm E, Pawliuk R, Wariaro D, Post MJ, Wahlberg E, Leboulch P and Cao Y: Angiogenic synergism, vascular stability and improvement of hind-limb ischemia by a combination of PDGF-BB and FGF-2. Nat Med 9: 604-613, 2003.

17. Kapranov P, Willingham AT and Gingeras TR: Genome-wide transcription and the implications for genomic organization. Nat Rev Genet 8: 413-423, 2007.

18. Wapinski $\mathrm{O}$ and Chang HY: Long noncoding RNAs and human disease. Trends cell Biol 21: 354-361, 2011.

19. Muers M: RNA: Genome-wide views of long non-coding RNAs. Nat Rev Genet 12: 742, 2011

20. Yang JH, Li JH, Shao P, Zhou H, Chen YQ and Qu LH: StarBase: A database for exploring microRNA-mRNA interaction maps from Argonaute CLIP-Seq and Degradome-Seq data. Nucleic Acids Res 39: D202-D209, 2011.

21. Yang JH, Li JH, Jiang S, Zhou H and Qu LH: ChIPBase: A database for decoding the transcriptional regulation of long non-coding RNA and microRNA genes from ChIP-Seq data. Nucleic Acids Res (Database Issue) 41: D177-D178, 2013.

22. Volders PJ, Helsens K, Wang X, Menten B, Martens L, Gevaert K, Vandesompele J and Mestdagh P: LNCipedia: A database for annotated human IncRNA transcript sequences and structures. Nucleic Acids Res (Database Issue) 41: D246-D251, 2013.
23. Amaral PP, Clark MB, Gascoigne DK, Dinger ME and Mattick JS: lncRNAdb: A reference database for long noncoding RNAs. Nucleic Acids Res (Database Issue) 39: D146-D151, 2011.

24. Bu D, Yu K, Sun S, Xie C, Skogerbø G, Miao R, Xiao H, Liao Q, Luo H, Zhao G, et al: NONCODE v3.0: Integrative annotation of long noncoding RNAs. Nucleic Acids Res (Database Issue) 40: D210-D215, 2012.

25. Dinger ME, Pang KC, Mercer TR, Crowe ML, Grimmond SM and Mattick JS: NRED: A database of long noncoding RNA expression. Nucleic Acids Res (Database Issue) 37: D122-D126, 2009.

26. Self SG, Longton G, Kopecky KJ and Liang KY. On estimating HLA/disease association with application to a study of aplastic anemia. Biometrics 47: 53-61, 1991.

27. Baldwin JL, Storb R, Thomas ED and Mannik M: Bone marrow transplantation in patients with gold-induced marrow aplasia. Arthritis Rheum 20: 1043-1048, 1997.

28. Zhang T, Wang J, Zhou X, Liang R, Bai Q, Yang L, Gu H, Gao G, Dong B, Zhu $\mathrm{H}$ and Chen X: Increased Expression of TIGIT on CD4+ T cells ameliorates immune-mediated bone marrow failure of aplastic anemia. J Cell Biochem 115: 1918-1927, 2014.

29. Wang M, Nie N, Feng S, Shi J, Ge M, Li X, Shao Y, Huang J and Zheng Y: The polymorphisms of human leukocyte antigen loci may contribute to the susceptibility and severity of severe aplastic anemia in Chinese patients. Hum Immunol 75: 867-872, 2014.

30. Eom YW, Oh JE, Lee JI, Baik SK, Rhee KJ, Shin HC, Kim YM, Ahn CM, Kong JH, Kim HS and Shim KY: The role of growth factors in maintenance of stemness in bone marrow-derived mesenchymal stem cells. Biochem Biophys Res Commun 445: 16-22, 2014.

31. Chen P, Zhang L, Weng T, Zhang S, Sun S, Chang M, Li Y, Zhang B and Zhang L: A Ser252Trp mutation in fibroblast growth factor receptor 2 (FGFR2) mimicking human Apert syndrome reveals an essential role for FGF signaling in the regulation of endochondral bone formation. PloS One 9: e87311, 2014.

32. Tian Y, Zhang X, Hao Y, Fang Z and He Y: Potential roles of abnormally expressed long noncoding RNA UCA1 and Malat-1 in metastasis of melanoma. Melanoma Res 24: 335-341, 2014.

33. Huang YS, Hsieh HY, Shih HM, Sytwu HK and Wu CC: Urinary $\mathrm{Xist}$ is a potential biomarker for membranous nephropathy. Biochem Biophys Res Commun 452: 415-421, 2014.

34. Sedaie Bonab A, Pouladi N, Hosseinpourfeizi MA, Ravanbakhsh Gavgani R, Dehghan R, Azarfam P, Montazeri V and Fakhrjou A: Single-strand conformational polymorphism analysis of a common single nucleotide variation in WRAP53 gene, rs2287499 and evaluating its association in relation to breast cancer risk and prognosis among Iranian-Azeri population. Med Oncol 31: 168, 2014.

35. Bilinovich SM, Davis CM, Morris DL, Ray LA, Prokop JW, Buchan GJ and Leeper TC: The C-terminal domain of SRA1p has a fold more similar to PRP18 than to an RRM and does not directly bind to the SRA1 RNA STR7 region. J Mol Biol 426: 1753-1765, 2014

36. Sciacca L, Cassarino MF, Genua M, Vigneri P, Giovanna Pennisi M, Malandrino P, Squatrito S, Pezzino V and Vigneri R: Biological effects of insulin and its analogs on cancer cells with different insulin family receptor expression. J Cell Physiol 229: 1817-1821, 2014

37. Chen H, Xin Y, Zhou L, Huang JM, Tao L, Cheng L and Tian J: Cisplatin and paclitaxel target significant long noncoding RNAs in laryngeal squamous cell carcinoma. Med Oncol 31: 246, 2014.

38. Deb S, Johansson I, Byrne D, Nilsson C, Investigators $\mathrm{K}$, Constable L, Fjällskog ML, Dobrovic A, Hedenfalk I and Fox SB: Nuclear HIF1A expression is strongly prognostic in sporadic but not familial male breast cancer. Mod Pathol 27: 1223-1230, 2014 